\title{
Correlation of Serum Homocysteine with Attention and Executive Functions in Patients with Type I Stable Bipolar Affective Disorder
}

\author{
Wan-Qiu Na ${ }^{1}$, Wei Su${ }^{1,}$, Ju-Shui Sun ${ }^{1}$, Hai-Zhi Chen ${ }^{1}$, Li-Na Wu ${ }^{1}$, Jian-Hua Li ${ }^{1}$ and Li-Feng Mei ${ }^{1}$ \\ 1 Department of Psychiatry, Huzhou Third Municipal Hospital, Affiliated Hospital of Huzhou University, Huzhou, China
}

* Corresponding author: Wei Su, Department of Psychiatry, Huzhou Third Municipal Hospital, Affiliated Hospital of Huzhou University, Huzhou, China. Tel: +8605722290542; Fax: +8605722023856; Email: suwei_yui@163.com

Received 2020 0ctober 15; Revised 2020 November 01; Accepted 2020 November 07.

\begin{abstract}
Background: Bipolar affective disorder (BPAD) is a severe and persistent mental illness defined by the presence of recurrent mood episodes. Several studies have shown that the levels of homocysteine (Hcy) were elevated in BPAD patients, and high levels of peripheral Hcy have been associated with worse cognitive performance.

Objectives: The current study aimed to examine the correlation of the level of Hcy in the serum with attention and executive functions in patients suffering from type I stable BPAD (BPAD I).

Methods: The present cross-sectional study was conducted on 170 participants in Huzhou, Zhejiang province, China, within July 2016 to December 2017. The subjects were divided into patients with schizophrenia $(n=100)$ as the study group and healthy volunteers $(n=70)$ as the control group. The Hcy and folic acid levels of fasting ulnar vein serum were determined using chemiluminescent microparticle immunoassay. Patient attention was evaluated by the Trail Making Test A (TMTA), and executive functions were assessed using the Trail Making Test B (TMTB) and Stroop Color and Word Test (SCWT).

Results: The mean value of serum Hcy was significantly higher in the study group than that reported for the control group $(19.01 \pm 5.83$ and 11.40 $\pm 4.62 ; \mathrm{P}<0.001)$. The results of several tests of attention $(59.39 \pm 19.27$ and $53.26 \pm 14.82 ; \mathrm{P}=0.027)$ and executive functions $(123.75 \pm 40.60$ and $107.52 \pm 29.31 ; \mathrm{P}=0.013)$ were poorer in the study group. The serum Hcy value positively correlated with the disturbance variables of the TMTA, TMTB, and Trail Making Test. However, the duration of the Stroop-C test (SCT) and SCWT negatively correlated with the correct number of the SCT and SCWT.

Conclusion: The increase in the level of serum Hcy closely correlated with the impairment of attention and executive functions in patients suffering from stable BPAD I.
\end{abstract}

Keywords: Attention, Bipolar affective disorder, Executive function, Folate, Homocysteine

\section{Background}

Bipolar affective disorder (BPAD) is a severe and persistent mental illness defined by the presence of recurrent mood episodes (1). The BPAD is divided into two subtypes in the fourth edition of the Diagnostic and Statistical Manual of Mental Disorders (DSM-IV). The BPAD type I (BPAD I) refers to a manic or mixed episode and major depressive episode; however, BPAD type II refers to a mild mania and major depressive episode. Cognitive impairment occurs in both the acute and stable stages of BPAD patients (1-2). Furthermore, the impairment of BPAD I is more serious than the impairment of BPAD II (3). In particular, cognitive impairment in the stable stage of BPAD I suggests poor prognosis (4), and this is the main reason for no restoration of normal social function in the remission stage (5).

Attention and executive functions are important components of cognitive function, which can affect the final recovery and long-term prognosis of patients (6). Homocysteine (Hcy) has a neurotoxic effect on neuronal apoptosis (7), which closely correlated with cognitive impairment in many diseases, such as dementia, depression, and schizophrenia (8-10). Several studies have shown that the levels of Hcy were elevated in BPAD patients, and high levels of peripheral Hcy have been associated with worse cognitive performance (11-13).

In 2008, Dittmann et al. reported that elevated Hcy levels might have a negative impact on verbal learning, delayed memory, and executive function in BPAD patients (11). In another study, Osher et al. obtained a similar result. They confirmed that in BPAD patients, higher Hcy levels were associated with poorer performance in some neuropsychological tests (13). A meta-analysis conducted by Salagre et al. evaluated nine cross-sectional studies with 1,547 participants. The results of the aforementioned study showed that Hcy levels were elevated in patients with BPAD during mania and euthymia, suggesting that peripheral Hcy could be considered a potential biomarker in BPAD (14).

\section{Objectives}

To date, no study has reported the prevalence of Hcy in Chinese patients suffering from stable BPAD I. Therefore, the present study aimed to investigate the relationship between the level of serum Hcy and 
impairment of attention and executive functions in patients with stable BPAD I.

\section{Methods}

\subsection{Subjects}

This cross-sectional study was conducted on 170 participants in Huzhou, Zhejiang province, China. Huzhou Third Municipal Hospital is a governmental specialized hospital with 900 beds. The Psychiatry Department is the major department of this hospital. A total of 100 outpatients or inpatients (including 50 males and 50 females) in the No. 3 People's Hospital of Huzhou were assigned to the study group within July 2016 and December 2017; however, 70 health workers of the hospital were assigned to the control group (including 35 males and 35 females).

Most $(85 \%)$ of the patients were from Huzhou. Only $15 \%$ of the patients were from the cities next to Huzhou, such as Jiaxin and Wenzhou. And all the health workers were natives of Huzhou. All the study subjects were right-handed, with no color blindness or weakness and no deafness or stuttering. Furthermore, the subjects were not related to each other and were able to independently complete the neuropsychological tests. The present study was approved by the Ethics Committee of No. 3 People's Hospital of Huzhou (code: 201602; approval date: 17 April 2016), and informed consent was obtained from all the patients.

\subsection{Inclusion and exclusion criteria}

The inclusion criteria of the present study were 1) the diagnostic criteria for BPAD I based on the DSM-IV, evaluated by two attending physicians (or more), 2) remission period of $\geq 3$ months, remission stage standard (i.e., Young Mania Rating Scale [YMRS] score of $<6$ and Hamilton Depression Rating Scale-17 (HAMD-17) score of $\leq 7$ (15), 3) age range of 16-60 years, (4) educational level of $>5$ years, (5) and written informed consent (by the patient or guardian) for the present study. In addition, the patients had a personal physician who could independently evaluate the relevant neuropsychological condition.

The exclusion criteria of the current study were 1) a history of substance abuse and dependence, 2) presence of cellular-associated disease in the brain, 3 ) reception of electroconvulsive therapy in the past year, 4) experience of serious suicide ideation and self-injury, 5) presence of severe physical diseases, including heart, liver, and kidney diseases, 6) pregnancy or lactation, and 7) mental retardation.

\subsection{Sociodemographic data}

The sociodemographic data of the patients in the present study, including gender, age, educational level, marital status, course of the disease, and family history, were collected using a self-made general condition questionnaire.

\subsection{Executive function assessment}

The neuropsychological evaluation scale is an effective method for testing the impairment of executive function. The Trail Making Test A (TMTA) was used to assess the selective attention of the subjects in the present study. The Trail Making Test B (TMTB) and Stroop Color and Word Test (SCWT) were mainly applied to test the executive function of the patients. All the participants received the attention and executive function evaluation. The test was performed in a quiet environment and lasted for 30-60 min. The evaluation tools were successively introduced according to the actual test sequence as follows:

1) The Trail Making Test (TMT) (16) consists of two parts (A and B), which was used for the evaluation of completion time. In both tests, if the completion time is shorter, the score is better. The TMT disturbance variable reflects the set-shift components of executive function to complete the difference of the time consumed between the TMTA and TMTB.

2) The SCWT (17) includes the Stroop-C test (SCT) and SCWT. Each card has 112 words for the assessment of the reading time and correct number.

All the symptom scales and executive functions were completed on the same day; however, the study subjects were allowed to rest between the assessments.

\subsection{Homocysteine and folate determination}

Serum Hcy and folic acid levels were simultaneously measured in the study and control groups. The blood was sampled at 8:00 a.m. the day after the enrollment. Ulnar vein blood ( $5 \mathrm{~mL}$ ) was collected on an empty stomach, and the blood samples were placed in a vacuum coagulation tube. Serum centrifugation was performed within $1 \mathrm{~h}$ at 3,000 rpm for 5-10 min (Allegra 64R Centrifuge, Beckman Coulter, Brea, California, the USA). The supernatant was aspirated and stored at $-70^{\circ} \mathrm{C}$ for subsequent tests. The levels of serum Hcy and folic acid were measured via a chemiluminescence kit (Abbott Laboratories, Illinois, the USA). Chemiluminescence microparticle immunoassay was performed on the ARCHITECT i2000 SR (Abbott Laboratories, Illinois, the USA). This operation was carried out according to the standard procedure.

\subsection{Statistical analysis}

The SPSS statistical software (version 20.0; IBM Corp., Armonk, New York, USA) was used to process the data. The measured data were expressed as mean \pm standard deviation. The enumerated data were expressed in percentage (\%). The $\mathrm{W}$-test was utilized to test the normality, and the F-test was used for the homogeneity test of variances. In addition, the t-test 
was employed for comparisons between the two groups. The nonparametric test was utilized to compare the mean of multiple samples not obeying the normal distribution or obeying the normal distribution but with an uneven variance. The counting data were used for the Chi-square test. Correlation analysis was based on the Pearsonrelated analysis or Spearman-related analysis. A pvalue of less than 0.05 was considered statistically significant.

\section{Results}

\subsection{Sociodemographic and clinical characteristics}

In this study, a total of 110 patients with BPAD I were screened within July 2016 and December 2017. Nevertheless, 10 patients did not meet the inclusion criteria and were ruled out. Finally, 100 patients were included in the study group of the present study. The course of the disease was within 18-55 months with a median of $27.54 \pm 6.32$ months. Seven patients had a positive family history of BPAD I. The control group comprised of 70 healthy subjects. The differences in age, educational level, and marital status between these two groups were not statistically significant $(\mathrm{P}>0.05)$. The YMRS and HAMD-17 scores were significantly higher in the study group than those reported for the control group $(\mathrm{P}<0.001$; Table 1$)$.

4.2. Comparison of the levels of serum Hcy and folic acid in addition to attention and executive functions

The serum Hcy level was significantly higher in the study group than that reported for the control group $(\mathrm{P}<0.001)$; however, the difference in the folic acid level was not statistically significant $(P>0.05)$. Furthermore, attention function and all evaluated executive functions were significantly poorer in the study group than those reported for the control group $(\mathrm{P}<0.05$; Table 2$)$.

\begin{tabular}{|c|c|c|c|c|}
\hline Index & $\begin{array}{l}\text { Study group } \\
(n=100)\end{array}$ & $\begin{array}{c}\text { Control group } \\
(n=70)\end{array}$ & $\mathrm{t} / \chi^{2}$ & P-value \\
\hline Age (year) & $31.19 \pm 10.73$ & $32.13 \pm 11.17$ & 0.553 & 0.581 \\
\hline Duration of education & $10.17 \pm 3.59$ & $11.08 \pm 4.12$ & 1.530 & 0.128 \\
\hline Spouse (Yes/No) & $74 / 26$ & $48 / 22$ & 0.599 & 0.439 \\
\hline Course of disease (month) & $27.54 \pm 6.32$ & -- & -- & -- \\
\hline Family history & 7 & -- & -- & -- \\
\hline Young Mania Rating Scale & $1.82 \pm 1.10$ & $0.48 \pm 0.79$ & 8.734 & 0.000 \\
\hline Hamilton Depression Rating Scale-17 & $2.28 \pm 2.35$ & $0.81 \pm 1.06$ & 4.894 & 0.000 \\
\hline
\end{tabular}

Table 2. Comparison of serum homocysteine and folic acid levels in addition to attention and executive functions between study and control groups

\begin{tabular}{|c|c|c|c|c|c|}
\hline Index & Unit & $\begin{array}{c}\text { Study group } \\
(n=100)\end{array}$ & $\begin{array}{c}\text { Control group } \\
(n=70)\end{array}$ & $\mathbf{T} / \mathbf{Z}$ & P-value \\
\hline Homocysteine & umol/L & $19.01 \pm 5.83$ & $11.40 \pm 4.62$ & 9.100 & $<0.001$ \\
\hline Folic acid & $\mathrm{ng} / \mathrm{L}$ & $6.53 \pm 2.81$ & $7.32 \pm 2.55$ & 1.873 & 0.063 \\
\hline TMTA & $\mathrm{Sec}$ & $59.39 \pm 19.27$ & $53.26 \pm 14.82$ & 2.238 & 0.027 \\
\hline TMTB & $\mathrm{Sec}$ & $123.75 \pm 40.60$ & $107.52 \pm 29.31$ & 2.509 & 0.013 \\
\hline TMT interference & $\mathrm{Sec}$ & $62.41 \pm 29.60$ & $53.85 \pm 22.57$ & 2.137 & 0.033 \\
\hline SCT & Amount & $106.80 \pm 7.37$ & $110.35 \pm 3.24$ & 3.780 & $<0.001$ \\
\hline SCT & Sec & $70.45 \pm 22.52$ & $62.82 \pm 15.03$ & 2.474 & 0.014 \\
\hline SCWT & Amount & $78.17 \pm 28.85$ & $87.30 \pm 20.91$ & 2.263 & 0.025 \\
\hline SCWT & $\mathrm{Sec}$ & $170.83 \pm 59.73$ & $151.46 \pm 40.92$ & 2.577 & 0.011 \\
\hline
\end{tabular}

TMTA: Trail Making Test A; TMTB: Trail Making Test B; TMT: Trail Making Test; SCT: Stroop-C test; SCWT: Stroop Color and Word Test

4.3. Correlation analysis of the levels of serum Hcy and folic acid with attention and executive functions in the study group

A Spearman correlation analysis was carried out for serum Hcy and folic acid levels, age, duration of education, course of the disease, TMTA, TMTB, TMT (TMTB-TMTA), SCT, and SCWT in the study group. The level of serum Hcy significantly and positively correlated $(\mathrm{P}<0.05)$ with the duration of the TMTA and disturbance variable of the TMT. Moreover, the level of serum Hcy significantly and positively correlated $(\mathrm{P}<0.01)$ with the duration of the TMTB, SCT, and SCWT. The level of serum Hcy also significantly and negatively correlated $(\mathrm{P}<0.01)$ with the correct number of the SCT and SCWT. However, the age, course of the disease, duration of education, and level of serum folic acid in the study group did not correlate $(\mathrm{P}>0.05)$ with the disturbance variables of the TMTA, TMTB, TMT, SCT, and SCWT (Table 3).

\section{Discussion}

The obtained results of the present study revealed that the level of serum Hcy was higher in stable BPAD I in the study group than that reported for the control group; however, the difference in folic acid level was not statistically significant. The results of various tests of attention and executive functions were 


\begin{tabular}{|c|c|c|c|c|c|c|c|}
\hline Index & TMTA & TMTB & TMT interference & SCT & SCTs & SCWT & SCWTs \\
\hline \multirow{2}{*}{ Age (year) } & 0.078 & 0.009 & 0.113 & -0.033 & 0.278 & -0.037 & 0.105 \\
\hline & 0.599 & 0.943 & 0.487 & 0.840 & 0.069 & 0.822 & 0.263 \\
\hline \multirow{2}{*}{ Course of disease } & 0.093 & 0.271 & 0.215 & -0.126 & 0.252 & -0.172 & 0.031 \\
\hline & 0.570 & 0.091 & 0.184 & 0.438 & 0.117 & 0.302 & 0.879 \\
\hline \multirow{2}{*}{ Duration of education } & 0.102 & 0.008 & 0.073 & -0.237 & 0.172 & -0.064 & 0.203 \\
\hline & 0.530 & 0.854 & 0.653 & 0.141 & 0.288 & 0.696 & 0.178 \\
\hline \multirow{2}{*}{ Homocysteine } & $0.332^{*}$ & $0.408^{* *}$ & $0.389^{*}$ & $-0.603^{* *}$ & $0.572^{* *}$ & $-0.577^{* *}$ & $0.642^{* *}$ \\
\hline & 0.036 & 0.007 & 0.030 & 0.000 & 0.000 & 0.000 & 0.000 \\
\hline \multirow{2}{*}{ Folic acid } & 0.024 & 0.087 & 0.052 & -0.028 & 0.157 & -0.172 & 0.166 \\
\hline & 0.637 & 0.537 & 0.689 & 0.836 & 0.300 & 0.299 & 0.306 \\
\hline
\end{tabular}

* $\mathrm{P}<0.05 ; * * \mathrm{P}<0.01$

TMTA: Trail Making Test A; TMTB: Trail Making Test B; TMT: Trail Making Test; SCT: Stroop-C test; SCWT: Stroop Color and Word Test Upper row regarded as the value of correlation coefficient $r$; lower row regarded as the p-value

statistically poorer in the study group than those reported for the control group. The serum Hcy value of patients in the study group positively correlated with the disturbance variables of the TMTA, TMTB, and TMT and duration of the SCT and SCWT; nevertheless, the serum Hcy value negatively correlated with the correct number of the SCT and SCWT. Nonetheless, the serum folic acid level, age, education level, and course of disease did not correlate with attention and executive functions.

Executive function is an advanced cognitive function of human beings and the ability of individuals to successfully engage in independent, purposeful, and self-responsible behaviors. This is coordinated with attention function and is an important component of cognitive function in patients with BPAD (18). The BPAD is a form of chronic mental disorder with a high recurrence rate and high disability (19). Patients' social functioning could be recovered if they could manage BPAD treatment by themselves, such as adhering to drug treatment, maintaining a good mood, and achieving optimal control of the disease. However, all these factors rely on the capability for attention skills and executive functions.

The findings of the current study demonstrated that the time of the TMT and CWT was prolonged; nevertheless, the correct number of CWT decreased. This finding suggested that the impairment of attention and executive functions is observed in patients with stable BPAD I, which is consistent with the results of previous studies $(20,21)$. In another study carried out by Mann et al., it was also revealed that the impairment of executive functions in BPAD patients persists and does not fully recover in the stable stage. The impairment of executive functions in stable BPAD patients might be also one of the inner phenotypes of the disease (22).

Neuroimaging studies have shown that brain volume, white matter fiber integrity, cerebral blood flow signals, and oxygen saturation in important brain areas are the related factors of executive function impairment in patients with BPAD (23). Dev et al. (24) also demonstrated that the bilateral anterior cingulate, bilateral dorsolateral prefrontal cortex, and cerebral blood flow levels of the right inferior parietal lobule positively correlated with reactive inhibition. However, it is required to carry out further studies to investigate the aforementioned finding.

The results of a study conducted by Dittmann et al. (11) indicated that the increase of serum Hcy levels in BPAD patients is often accompanied by a decrease in executive functions, which is consistent with the findings of the current study. Therefore, the serum Hcy level may be a biological risk factor for cognitive dysfunction. In the present study, the level of serum Hcy significantly increased in the study group, compared to that reported for the control group $(\mathrm{P}<0.05)$. The level of serum Hcy in the study group also positively correlated $(\mathrm{P}<0.05)$ with the duration of the TMT and CWT; nonetheless, it negatively correlated $(\mathrm{P}<0.05)$ with the correct number of the CWT. This finding suggests that the increase in serum Hcy in stable BPAD I patients may further damage the attention and executive functions $(\mathrm{P}<0.05)$. These results are similar to those of studies by Dias VV et al. (12) and Dittmann S et al. (11).

The Hcy is an important intermediate product in the metabolism of methionine. A high Hcy level has been shown to promote the self-oxidation of Hcy, destroy the normal redox state of neural cells, affect the redox signaling pathway, and aggravate the oxidative damage of endothelial cells and neurons (25). Furthermore, a high level of Hcy may induce indirect neurotoxicity, leading to the death of hippocampal neurons and cognitive impairment in patients. In the current study, the presence and correlation of high levels of serum Hcy and damage to attention and executive functions in stable BPAD I patients $(P<0.05)$ suggest a connection between Hcy metabolism and behavioral functions. Currently, the mechanism of serum Hcy in BPAD remains unclear and needs to be further studied.

In the present study, the difference in the level of serum folic in the stable BPAD I group was not statistically significant $(\mathrm{P}>0.05)$, compared to that of the control group, which did not correlate with the TMT and SCT $(\mathrm{P}>0.05)$. In addition, age, educational level, and course of the disease did not correlate with 
the TMT and SCT $(\mathrm{P}>0.05)$. This result suggests that the impairment of attention and executive functions in stable BPAD I patients does not correlate with serum folic acid level, age, educational level, and course of the disease, which is in line with the results of a study conducted by Normala et al. (26).

In the current study, it was observed that the damage to attention and executive functions in Chinese stable BPAD I patients correlated with the high level of Hcy. It is regarded as the novelty of the present study. However, the present study also had several limitations. Firstly, the present study only carried out a comparative investigation of the crosssectional data. Secondly, the present study was a single-center clinical study with a small sample size; therefore, it is required to carry out further studies with a larger sample size and multicenter clinical nature. Finally, further stratification was not carried out for the Hcy level, and drug analysis and grouping were not conducted in the present study; consequently, further investigation is still required in this regard.

\section{Conclusion}

The patients with stable BPAD I demonstrated a correlated and significant increase in their levels of serum Hcy and impairment of attention and executive functions.

\section{Acknowledgments}

The authors would like to acknowledge the hard and dedicated work of all the staff implementing the intervention and evaluation components of the study.

\section{Footnotes}

Authors' Contribution: Wan-Qiu Na and Wei Su conceived the idea and conceptualized the present study. Ju-Shui Sun, Hai-Zhi Chen, and $\mathrm{Li}-\mathrm{Na} \mathrm{Wu}$ collected the data. Jian-Hua $\mathrm{Li}$ and Li-Feng Mei analyzed the data. Wan-Qiu $\mathrm{Na}$ and Wei Su drafted the manuscript. Wan-Qiu Na and Wei Su reviewed the manuscript. All the authors read and approved the final draft.

Conflict of Interests: All the authors declared that there are no personal, financial, commercial, or academic conflicts of interest.

Ethical Approval: The present study was approved by the Ethics Committee of No. 3 People's Hospital of Huzhou (code: 201602; approval date: 17 April 2016).

Informed consent: Informed consent was obtained from all the patients.

\section{References}

1. Lee RS, Hermens DF, Scott J, Redoblado-Hodge MA, Naismith
$\mathrm{SL}$, Lagopoulos J, et al. A meta-analysis of neuropsychological functioning in first-episode bipolar disorders. J Psychiatr Res. 2014;57:1-11. doi: 10.1016/j.jpsychires.2014.06.019. [PubMed: 25016347].

2. Rosa AR, Magalhães PV, Czepielewski L, Sulzbach MV, Goi PD, Vieta E, et al. F. Clinical staging in bipolar disorder: focus on cognition and functioning. J Clin Psychiatry. 2014;75(5):e4506. doi: 10.4088/JCP.13m08625. [PubMed: 24922497].

3. Galimberti C, Bosi MF, Caricasole V, Zanello R, Dell'Osso B, Viganò CA. Using network analysis to explore cognitive domains in patients with unipolar versus bipolar depression: a prospective naturalistic study. CNS Spectr. 2020;25(3):380-91. doi: 10.1017/S1092852919000968. [PubMed: 31060642].

4. de Filippis R, Aloi M, Bruni A, Gaetano R, Segura-Garcia C, De Fazio P. Bipolar disorder and obsessive compulsive disorder: The comorbidity does not further impair the neurocognitive profile. J Affect Disord. 2018;235:1-6. doi: 10.1016/j.jad. 2018.03.010. [PubMed: 29627704].

5. Anaya C, Torrent C, Caballero FF, Vieta E, Bonnin Cdel M, Ayuso-Mateos JL; CIBERSAM Functional Remediation Group. Cognitive reserve in bipolar disorder: relation to cognition, psychosocial functioning and quality of life. Acta Psychiatr Scand. 2016;133(5):386-98. doi: 10.1111/acps.12535. [PubMed: 26719018].

6. Schwarz K, Moessnang C, Schweiger JI, Baumeister S, Plichta MM, Brandeis D, et al. Transdiagnostic prediction of affective, cognitive, and social function through brain reward anticipation in schizophrenia, bipolar disorder, major depression, and autism spectrum diagnoses. Schizophr Bull. 2020;46(3):592-602. doi: 10.1093/schbul/sbz075. [PubMed: 31586408].

7. Tian X, Gong L, Jin A, Wang Y, Zhou X, Tan Y. E3 ubiquitin ligase siah-1 nuclear accumulation is critical for homocysteine-induced impairment of C6 astroglioma cells. Mol Med Rep. 2019; 20(3):2227-35. doi: 10.3892/mmr.2019.10449. [PubMed: 31322210].

8. El-Missiry MA, ElKomy MA, Othman AI, AbouEl-Ezz AM. Punicalagin ameliorates the elevation of plasma homocysteine, amyloid- $\beta$, TNF- $\alpha$ and apoptosis by advocating antioxidants and modulating apoptotic mediator proteins in brain. Biomed Pharmacother. 2018;102:472-80. doi: 10.1016/j.biopha.2018.03.096. [PubMed: 29579708].

9. Pierozan P, Biasibetti-Brendler H, Schmitz F, Ferreira F, Netto CA, Wyse ATS. Synergistic toxicity of the neurometabolites quinolinic acid and homocysteine in cortical neurons and astrocytes: implications in alzheimer's disease. Neurotox Res. 2018;34(1):147-63. doi: 10.1007/s12640-017-9834-6. [PubMed: 29124681].

10. Karkhah A, Ataee R, Ataie A. Morphine pre- and postconditioning exacerbates apoptosis in rat hippocampus cells in a model of homocysteine-induced oxidative stress. Biomed Rep. 2017;7(4):309-13. doi: 10.3892/br.2017.962. [PubMed: 28928969].

11. Dittmann S, Seemüller F, Grunze HC, Schwarz MJ, Zach J, Fast K, et al.The impact of homocysteine levels on cognition in euthymic bipolar patients: a cross-sectional study. J Clin Psychiatry. 2008;69(6):899-906. doi: 10.4088/jcp.v69n0603. [PubMed: 18399726].

12. Dias VV, Brissos S, Cardoso C, Andreazza AC, Kapczinski F. Serum homocysteine levels and cognitive functioning in euthymic bipolar patients. J Affect Disord. 2009;113(3):285-90. doi: 10.1016/j.jad.2008.05.011. [PubMed: 18579214].

13. Osher Y, Bersudsky Y, Silver H, Sela BA, Belmaker RH. Neuropsychological correlates of homocysteine levels in euthymic bipolar patients. J Affect Disord. 2008;105(1-3):22933. doi: 10.1016/j.jad.2007.04.005. [PubMed: 17490752].

14. Salagre E, Vizuete AF, Leite M, Brownstein DJ, McGuinness A, Jacka F, et al. Homocysteine as a peripheral biomarker in bipolar disorder: A meta-analysis. Eur Psychiatry. 2017;43:8191. doi: 10.1016/j.eurpsy.2017.02.482. [PubMed: 28371745].

15. Weiner L, Doignon-Camus N, Bertschy G, Giersch A. Thought and language disturbance in bipolar disorder quantified via process-oriented verbal fluency measures. Sci Rep. 2019; 9(1):14282. doi: 10.1038/s41598-019-50818-5. [PubMed: 
31582814].

16. Fontana E, Mandolini GM, Delvecchio G, Bressi C, Soares JC, Brambilla P. Intravenous valproate in the treatment of acute manic episode in bipolar disorder: A review. J Affect Disord. 2020;260:738-43. doi: 10.1016/j.jad.2019.08.071. [PubMed: 31581039].

17. Alosaimi FD, AlAteeq DA, Bin Hussain SI, Alosaimi FD, AlAteeq DA, Bin Hussain SI, et al. Public awareness, beliefs, and attitudes toward bipolar disorder in saudi arabia. Neuropsychiatr Dis Treat. 2019;15:2809-18. doi: 10.2147/NDT.S209037. [PubMed: 31579220].

18. Nguyen TT, Kovacevic S, Dev SI, Lu K, Liu TT, Eyler LT. Dynamic functional connectivity in bipolar disorder is associated with executive function and processing speed: A preliminary study. Neuropsychology. 2017;31(1):73-83. doi: 10.1037/neu0000317. [PubMed: 27775400].

19. Whiteford HA, Degenhardt L, Rehm J, Baxter AJ, Ferrari AJ, Erskine HE, et al. Global burden of disease attributable to mental and substance use disorders: findings from the global burden of disease study 2010. Lancet. 2013;382(9904): 1575-86. doi: 10.1016/S0140-6736(13)61611-6. [PubMed: 23993280].

20. Newton DF, Naiberg MR, Andreazza AC, Scola G, Dickstein DP, Goldstein BI. Association of lipid peroxidation and brainderived neurotrophic factor with executive function in adolescent bipolar disorder. Psychopharmacology (Berl). 2017; 234(4): 647-56. doi: 10.1007/s00213-016-4500-x. [PubMed:
27957714].

21. Bora E, Hıdıroğlu C, Özerdem A, Kaçar ÖF, Sarısoy G, Civil Arslan F, et al. Executive dysfunction and cognitive subgroups in a large sample of euthymic patients with bipolar disorder. Eur Neuropsychopharmacol. 2016;26(8):1338-47. doi: 10.1016/j.euroneuro.2016.04.002. [PubMed: 27139077].

22. Mann-Wrobel MC, Carreno JT, Dickinson D. Meta-analysis of neuropsychological functioning in euthymic bipolar disorder: an update and investigation of moderator variables. Bipolar Disord. 2011;13(4):334-42. doi: 10.1111/j.1399-5618.2011.00935.x. [PubMed: 21843273].

23. Strakowski SM, DelBello MP, Adler C, Cecil DM, Sax KW. Neuroimaging in bipolar disorder. Bipolar disord. 2000;2(3 Pt 1):148-64. doi: 10.1034/j.1399-5618.2000.020302.x. [PubMed: 11256682].

24. Dev SI, McKenna BS, Sutherland AN, Shin DD, Liu TT, Wierenga $\mathrm{CE}$, et al. Increased cerebral blood flow associated with better response inhibition in bipolar disorder. J Int Neuropsycbol Soc. 2015;21(2):105-15. doi: 10.1017/S135561771400112X. [PubMed: 25771682].

25. Zou CG, Banerjee R. Homocysteine and redox signaling. Antioxid Redox Signal. 2005;7(5-6):547-59. doi: 10.1089/ars. 2005.7.547. [PubMed: 15890000].

26. Normala I, Abdul HA, Azlin B, Nik Ruzyanei NJ, Hazli Z, Shah SA. Executive function and attention span in euthymic patients with bipolar 1 disorder. Med J Malaysia. 2010;65(3):199-203. [PubMed: 21939168]. 